\title{
MODELO DE EVALUACIÓN DE SEGURIDAD VIAL PARA PAÍSES CON ESCASEZ DE INFORMACIÓN ESTRUCTURADA
}

\author{
Fabricio Espinoza Molina \\ Ingeniero, estudiante de Doctorado en la UPM, Docente - Investigador, de la Universidad \\ Politécnica Salesiana, Cuenca, Ecuador. fespinoza@ups.edu.ec \\ Blanca Arenas Ramírez \\ Profesor Contratado Doctor. ETSI Industriales, Directora de la Unidad de Estudios de \\ Transporte e Impacto Medioambiental del Automóvil de (INSIA), España, \\ barenas@etsii.upm.es

\section{Francisco Aparicio Izquierdo} \\ Profesor Emérito de la UPM. Presidente del Instituto Universitario de Investigación del \\ Automóvil (INSIA), francisco.aparicio@upm.es
}

\begin{abstract}
RESUMEN
La información para analizar la situación de la seguridad vial de un país tiene que ser confiable, estructurada y sólida, como requisito fundamental para evaluar los factores de influencia en los accidentes, víctimas, etc. En los países emergentes la información es desordenada, de baja calidad, incompleta, y en ocasiones nula. Con frecuencia, la que existe no está estructurada de manera adecuada y su utilidad es limitada y escasa para el trabajo de investigación, análisis y diagnóstico de la situación y la adopción de medidas de mejora en seguridad vial, adaptadas a la situación de cada país. El objetivo es realizar una evaluación de la seguridad vial en los países de América del Sur definiendo para un futuro un desarrollo de una metodología de evaluación de seguridad vial para países emergentes con escasez de información estructurada, la cual es una estrategia de gran valor para ayudar a definir y evaluar las medidas que se implementaran a futuro para conseguir los objetivos y metas del Decenio de Acción para la Seguridad Vial 2011-2020 en relación a la reducción del número de muertes en las carreteras, en el caso de los países latinoamericanos. Materiales y métodos se revisaron exhaustivamente las bases de datos científicas como Scopus y Scielo y los planes de seguridad vial e informes que poseen las distintas entidades relacionadas con la seguridad vial de los países de América del Sur, para la sistematización de la información se aplicó la metodología del meta-análisis. El resultado se evidencia la falta de investigación científica en accidentes de tránsito, además se identifica sus debilidades, fortalezas y líneas de acción sugeridas a ser implementadas en los planes de seguridad vial. Conclusión la información disponible presenta dificultades para reconocer la real situación la cual ocasiona enormes problemas para establecer comparaciones entre países dentro de la región América del Sur.
\end{abstract}




\section{INTRODUCION}

Los accidentes de tránsito son un problema que se presenta en todos los países del mundo, son causantes de graves lesiones, miles de decesos y se ha convertido en un problema de salud pública a nivel mundial, las lesiones causadas por el tránsito según " (Organizacion Mundial de la Salud, 2013) son la octava causa mundial de muerte, y la primera entre los jóvenes de 15 a 29 años". Las tendencias actuales indican que, si no se toman medidas urgentes, los accidentes de tránsito se convertirán en 2030 en la quinta causa de muerte.

En marzo de 2010, la resolución 64/255 de la "Asamblea General de las Naciones Unidas proclamó el periodo 2011- 2020 como el Decenio de Acción para la Seguridad Vial (ONU, 2010)" con el objetivo de estabilizar y, posteriormente, reducir las cifras previstas de víctimas mortales en accidentes de tránsito en todo el mundo.

\subsection{La accidentalidad en Iberoamérica}

En la Figura 1 se muestra la comparativa de fallecidos por 100.000 habitantes de los países de Latinoamérica, del "sexto informe Iberoamericano de Seguridad Vial (OISEVI, 2015)". Los niveles del indicador en la mayoría de los países de la región son elevados, y demandan grandes esfuerzos para la mejora de éste y otros índices de accidentabilidad.

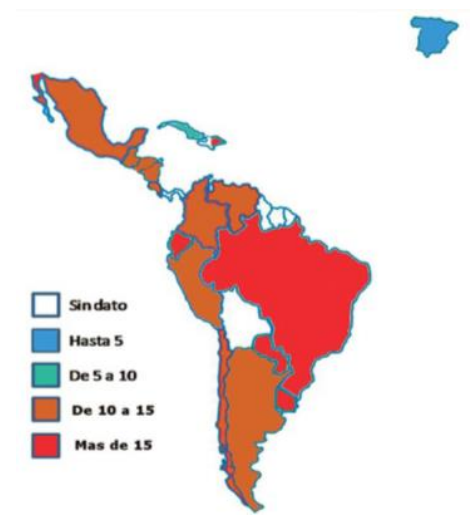

Fig. 1 - Mapa tasa de fallecidos cada 100.000 habitantes años 2000 y 2013 Fuente: VI Informe OISEVI. Pag. 24

\section{FUNDAMENTACION}

La Seguridad Vial (SV) es el conjunto de acciones y mecanismos que garantizan el buen funcionamiento de la circulación del tránsito, mediante la utilización de conocimientos técnicos y científicos así como la aplicación de leyes, reglamentos y disposiciones que operen sobre el comportamiento humano, bien sea en el papel de peatón, pasajero o conductor, a fin de usar correctamente la vía pública previniendo los accidentes de tránsito.

\subsection{Factores riesgo}

Entendemos por factor de riesgo todo aquel elemento, fenómeno, condición, circunstancia o acción humana que incrementa la probabilidad de ocurrencia de un accidente. Los distintos factores implicados en la accidentalidad tienen un peso diferencial en función de las condiciones concretas operativas, es decir, para un determinado accidente habrá algunos factores más importantes que otros. Los distintos factores implicados en la accidentalidad según la estadística atribuyen al factor humano una influencia como causa directa o indirecta de los accidentes superior al $90 \%$ al factor medio 10 y el $30 \%$ y al factor vehículo entre un 4 y un $10 \%$. Estos valores, meramente orientativos dependen de los contextos nacionales. Existe, no obstante coincidencia en que el factor humano es el de mayor influencia y el 
vehículo el de influencia relativa inferior. Esta puede ser la causa por los que se han centrado muchos esfuerzos en el estudio del factor humano para la prevención los accidentes de tránsito.

Entre los modelos propuestos para sistematizar y analizar los factores de influencia sobre los accidentes de tránsito figura el de (Haddon, 1972) que concretó el proceso del accidente vehicular en tres fases: previa a la colisión, durante la colisión y después de la colisión y los factores de influencia en cuadrados en "tres componentes: a) comportamiento humano, b) vehículo y equipamiento, c) vías y el entorno, formando una matriz con nueve campos que permite el análisis de los accidentes de tránsito" por medio de la interrelaciones de uno o más factores asociadas a las anteriores componentes, en las diferentes etapas del proceso de ocurrencia del accidente.

Existen aportes a la seguridad vial como el que se proponen agregar un cuarto elemento como complemento a la matriz de Haddon, y es el concepto de exposición, que permite contemplar la probabilidad de un evento o riesgo potencial por distancia recorrida o por unidad de tiempo determinada, " (Sivak \& Tsimhoni, 2008). Los autores argumentan que incluir el factor de exposición en la seguridad vial, contribuye a mejorar la efectividad de las decisiones técnicas".

Elvik, R. postula que es indispensable observar los resultados de las encuestas de consultas y las estadísticas de eventos de tránsito para tener una medida de la exposición al riesgo de un grupo específico poblacional, y que estos resultados deben analizarse a la luz del problema que se piensa resolver (Elvik, 2008).

Otro enfoque sobre el riesgo es considerar al factor humano un tanto separada del conductismo y psicóloga, para incorporar en el análisis de la seguridad vial a las personas en su calidad de conductores, y para tener en cuenta el control que haría la sociedad de su forma de actuar en las carreteras y calles (Izquierdo \& Torres, 2009).

\subsection{Problema de la información seguridad vial en América del Sur}

Hasta hace poco tiempo, la seguridad vial y la imperiosa necesidad de mejorarla para reducir el número de accidente y víctimas, no ha figurado entre las prioridades de la mayoría de los países de américa del sur y ello justifica que no se hayan establecido las estructuras, los métodos y los medios necesarios para contar con un sistema de información suficientemente desarrollado acerca de los accidentes, sus tipos, factores de influencia, víctima y otros daños ocasionados por los mismos. Actualmente el problema de sistematizar los datos de accidentes de tránsito en la región de América del Sur y el problema de la información está siendo encarada en algunos países, como se indica a continuación.

Argentina implementó el Formulario Estadístico Único (FEU) en el año 2010, como parte de un sistema uniforme de recolección de datos sobre siniestros viales de tal manera que le permite incorporar los registros individuales en una base de datos nacional.

En Brasil, los datos sobre las muertes por accidentes de tráfico están incluidos en el Sistema de Información (SIM) bajo la responsabilidad del Ministerio de Salud. Dicha base anual pasa por un proceso de revisión de datos, corrección y calificación.

Chile ha actualizado la ficha de recolección de datos de accidentes, para ello ha tomado en cuenta las recomendaciones de organismos internacionales (OMS, ONU), y la experiencia 
que han desarrollado países con altos estándares en tratamiento de datos (y que forman parte de OCDE, o aportan sus datos a la base IRTAD, entre otros).

Colombia reconoce que existen dificultades para la recolección y el análisis de la información que dé cuenta del estado de la Seguridad Vial, esto en gran parte a que las fuentes son diversas, algunas poco confiables y a que no existen protocolos estandarizados que definan una política para la recolección de datos.

En Ecuador la ANT trabaja actualmente en el Sistema de Levantamiento de Información de Siniestros de Tránsito (SILIST), a la fecha no se tiene conocimiento de su implementación para contar con una base de datos general que posibilite su análisis.

En Venezuela los datos en materia de siniestros son recabados por las direcciones de vigilancia del transporte terrestre de los cuerpos de policía nacional y municipal. El levantamiento de la información se realiza de forma manual, posteriormente es digitalizada en computadora para su procesamiento y sistematización, presentándose el riesgo de pérdida de exactitud en la información que se suministra.

En Paraguay, no existe un registro unificado de datos de seguridad vial. En el año 2004 se había conformado un observatorio interinstitucional para la toma y análisis de datos, pero no tuvo continuidad. El país cuenta con registros de siniestralidad vial de siete fuentes diversas.

En Perú la entidad encargada de la recolección de datos de accidentabilidad a nivel nacional es la Policía Nacional del Perú, la cual no efectúa la diferenciación de heridos graves y leves, debido a que no se especifica ésta información en su ficha de recolección en el momento de ocurrencia del accidente de tránsito.

\section{MATERIAL Y METODOS}

Para la realización del presente artículo se utilizó la metodología del meta - análisis, dividiendo en las siguientes fases.

Primero se parte de una revisión exhaustiva de las bases de datos científicas como: la Red de Revistas Científicas de América Latina y El Caribe, Web Of Science, ScienceDirect, Scopus y Scielo identificando solo los estudios publicados sobre evaluación de la seguridad vial en los países América del Sur, no se incluye a otros países del mundo.

La segunda fase se realizó mediante una búsqueda de los planes de seguridad vial en los respectivos sitios web específicos de los responsables del tránsito de los diez países de América del Sur PAS como son: Argentina, Bolivia, Brasil, Colombia, Chile, Ecuador, Paraguay, Perú, Uruguay y Venezuela, además se incluyó al Observatorio Iberoamericano de Seguridad Vial (OISEVI) y el diagnostico de seguridad vial realizado por la Asociación Española de Carretera y el Banco Interamericano de Desarrollo.

En la tercera fase, para aumentar el número de estudios e incluir aquellos no identificados en la estrategia de búsqueda inicial, se empleó términos como: driving questionnaire, driving scale behaviors and attitudes, road traffic crashes, y model assessment of road safety.

Los criterios de inclusión empleados para la selección de estudios fueron: 1) Fecha de publicación a partir de 1 de enero de 1990; 2) Población de estudio: conductores de vehículos automotores o peatones, 3) La selección de estudios se centró en los diez países de América del Sur; 4) El empleo de algún tipo de cuestionario (conductores y peatones) para evaluar la 
seguridad vial; 5) Idioma de publicación inglés, portugués y castellano.

\section{RESULTADOS Y DISCUSIÓN}

Lamentablemente no se identificaron artículos que cumplan con los criterios de inclusión del apartado metodológico, existiendo solo estudios en Argentina, Colombia y Perú, de comportamiento del conductor mediante la utilización de cuestionarios auto administrado. Las instituciones responsables de transito de algunos países como de Argentina, Colombia, Chile, Ecuador reportan en sus sitios web estudios del tipo observacional respecto al uso de casco o cinturón de seguridad del conductor, lo cual confirma la escasez de estudios, proyectos en materia de investigación científica de accidentes de tránsito en la región de América del Sur siendo su principal origen el problema de la información.

Según la “(Organización Panamericana de la Salud, 2009) realizar una comparación de la seguridad vial entre países resulta difícil, por las diferencias entre ellos". Por otra parte para tener un análisis completo y riguroso de la realidad accidentológica del país es necesario y de vital importancia contar con una definición de los indicadores de siniestralidad, vigilancia, base de datos, etc. Por ejemplo para la definición de persona fallecida en un accidente de tránsito se tiene varios criterios es así que Argentina, Chile y Uruguay los datos son computados a 30 días; Brasil y Colombia el dato computado al año; Bolivia, Ecuador, Paraguay, Perú y Venezuela tiene el dato registrado en el sitio o 24 horas, por lo que resulta difícil contar con una información armonizada tanto a nivel de región e internacionalmente. Es así que muchos de los análisis de la información aplican el "factor de corrector de 1,3" recomendado por la Organización Mundial de la Salud, (OMS) y la Conferencia Europea Ministros de Transporte, (CEMT) para poder relacionar el número de fallecidos con la población de un país y su parque automotor.

Un ejemplo del problema de la disponibilidad de la información se aprecia en la Tabla 1 según el sexto informe (OISEVI, 2015) con datos hasta el 2013 en el cual se observa que no todos cuenta con la información elemental.

\begin{tabular}{|c|c|c|c|c|c|c|c|}
\hline & 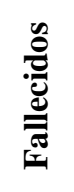 & 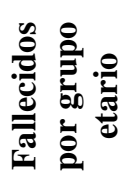 & 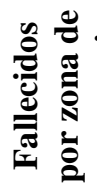 & 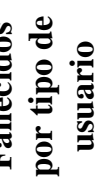 & $\begin{array}{l}\frac{5}{0} \\
\frac{\pi}{0} \\
\frac{\pi}{0}\end{array}$ & 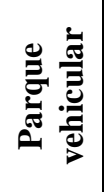 & 总 \\
\hline Argentina & $\mathrm{Si}$ & $\mathrm{Si}$ & $\mathrm{Si}$ & No & $\mathrm{Si}$ & $\mathrm{Si}$ & $\mathrm{Si}$ \\
\hline Bolivia & No & No & No & $\mathrm{No}$ & No & No & No \\
\hline Brasil & $\mathrm{Si}$ & No & No & $\mathrm{Si}$ & $\mathrm{Si}$ & $\mathrm{Si}$ & No \\
\hline Chile & $\mathrm{Si}$ & $\overline{\mathrm{Si}}$ & $\mathrm{Si}$ & $\mathrm{Si}$ & $\mathrm{Si}$ & $\overline{\mathrm{Si}}$ & No \\
\hline Colombia & $\mathrm{Si}$ & $\mathrm{Si}$ & No & $\mathrm{Si}$ & $\mathrm{Si}$ & $\mathrm{Si}$ & $\mathrm{Si}$ \\
\hline Ecuador & $\mathrm{Si}$ & No & $\mathrm{No}$ & No & $\mathrm{Si}$ & $\mathrm{Si}$ & No \\
\hline Paraguay & $\mathrm{Si}$ & $\mathrm{Si}$ & $\mathrm{Si}$ & $\mathrm{Si}$ & $\mathrm{Si}$ & $\mathrm{Si}$ & No \\
\hline Perú & $\mathrm{Si}$ & No & No & No & $\mathrm{Si}$ & $\mathrm{Si}$ & $\mathrm{Si}$ \\
\hline Uruguay & $\mathrm{Si}$ & $\mathrm{Si}$ & $\mathrm{Si}$ & $\mathrm{Si}$ & $\mathrm{Si}$ & $\mathrm{Si}$ & $\mathrm{Si}$ \\
\hline Venezuela & $\mathrm{Si}$ & No & No & No & $\mathrm{Si}$ & No & $\mathrm{No}$ \\
\hline
\end{tabular}

Tabla 1 - Disponibilidad de datos de accidentabilidad de los Países América Sur Elaboración propia

El diagnóstico realizado por la Asociación Española de Carretera y el Banco Interamericano de Desarrollo de la evaluación de la seguridad vial se basó en el análisis de los Planes Nacionales de Seguridad Vial (PNSV) de los países América del Sur y se resume en la Tabla 2. El objetivo de este estudio fue identificar las debilidades que presentan en cuanto a la 
información en la seguridad vial. Argentina, Colombia y Chile no presentan una deficiencia en la recopilación de datos de siniestralidad y análisis posterior y solo dos países Chile y Colombia no tienen deficiencias en cuanto información sobre la accidentalidad urbana incluyendo los usuarios vulnerables, nueve de diez países presentan problemas en el cumplimiento de las normas excepto Chile.

\begin{tabular}{|c|c|c|c|c|c|c|c|c|c|c|}
\hline Debilidades de seguridad vial & 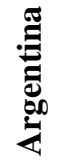 & "ृ: & فำ & $\frac{\frac{\pi}{0}}{\frac{0}{\tilde{\theta}}}$ & : & 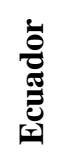 & 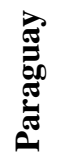 & 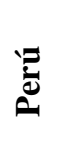 & 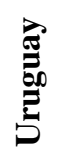 & 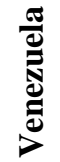 \\
\hline $\begin{array}{l}\text { Deficiencias en cuanto a la recopilación de } \\
\text { datos de siniestralidad y análisis posterior }\end{array}$ & & $\mathrm{X}$ & $\mathrm{X}$ & & & $\mathrm{X}$ & $\mathrm{X}$ & $X$ & $\mathrm{X}$ & $\mathrm{X}$ \\
\hline $\begin{array}{l}\text { Deficiencias en cuanto información sobre la } \\
\text { accidentalidad urbana incluyendo los usuarios } \\
\text { vulnerables }\end{array}$ & $\mathrm{X}$ & $\mathrm{X}$ & $X$ & & & $\mathrm{X}$ & $\mathrm{X}$ & $X$ & $X$ & $X$ \\
\hline $\begin{array}{l}\text { Escaso control del cumplimiento de las } \\
\text { normas }\end{array}$ & $\mathrm{X}$ & $\mathrm{X}$ & $\mathrm{X}$ & $\mathrm{X}$ & & $\mathrm{X}$ & $\mathrm{X}$ & $\mathrm{X}$ & $\mathrm{X}$ & $\mathrm{X}$ \\
\hline $\begin{array}{lllll}\begin{array}{l}\text { Necesidad de } \\
\text { formación vial }\end{array} & & & & \\
\end{array}$ & & $\mathrm{X}$ & $\mathrm{X}$ & & & & $\mathrm{X}$ & & & $X$ \\
\hline $\begin{array}{l}\text { No se ha implantado de manera reglamentaria } \\
\text { las herramientas de mejora de la seguridad de } \\
\text { las infraestructuras (auditoria seguridad vial) }\end{array}$ & $X$ & $\mathrm{X}$ & $\mathrm{X}$ & & $\mathrm{X}$ & $\mathrm{X}$ & $\mathrm{X}$ & $\mathrm{X}$ & $X$ & $X$ \\
\hline $\begin{array}{l}\text { No existe una Agencia líder dedicada a la } \\
\text { seguridad vial }\end{array}$ & & & $X$ & & & $X$ & & & & $X$ \\
\hline $\begin{array}{l}\text { No se ha regulado el uso de dispositivos de } \\
\text { retención infantil }\end{array}$ & & & & $\mathrm{X}$ & & $\mathrm{X}$ & $\mathrm{X}$ & $\mathrm{X}$ & & \\
\hline
\end{tabular}

Tabla 2 - Debilidades de los países América Sur en seguridad vial al 2013. Elaboración propia

En este trabajo los planes de seguridad vial incluidos para el análisis fueron: "Plan Nacional de Seguridad Vial 2010 (Agencia Nacional de Seguridad Vial, 2010)", "Plan plurinacional de seguridad vial 2014-2018 (Evo, y otros, 2014)", "Plano nacional de redução de acidentes e segurança viária para a década 2011 -2020 (DENATRAN, 2011)", "Plan de acción Estratégico de SV 2011-2014 (CONASET, 2013)", "Plan Nacional de seguridad vial 20112021 (Ministerio de Transporte de Colombia, 2014)", "Plan nacional de seguridad vial (ANT, 2013)", "Plan nacional de seguridad vial 2013-2018 (Consejo Nacional de Seguridad Vial, 2013)", "Plan nacional de seguridad vial del Perú 2015-2024 (Ministro de Transportes y Comunicaciones, 2015)" y "Plan de seguridad vial 2008 - 2012 (Ministerio Transporte y Obras Públicas, 2008)" de los países de Argentina, Bolivia, Brasil, Chile, Colombia, Ecuador, Paraguay, Perú, Uruguay y Venezuela respectivamente se resume mediante la Tabla 3.

El análisis se realiza de acuerdo a los cinco pilares fundamentales según las recomendaciones dadas en el Plan Mundial para el Decenio de Acción para la Seguridad Vial 2011-2020. En relación a la gestión de la seguridad vial declarados en los PNSV, Bolivia, Brasil, Ecuador, Paraguay y Venezuela no cuentan con los indicadores de resultados y siniestralidad y Argentina, Colombia, Chile y Uruguay prevén la creación de un observatorio de la seguridad vial, el cual cumplirá funciones de planificación, evaluación y monitoreo de las estrategias de seguridad vial y realizar investigación de accidentes e informes. Solo Colombia y Paraguay han involucrado a organismos en la participación en la elaboración PNSV el cual muestra una deficiencia en la elaboración de su plan al no integrar a actores de la seguridad vial. 


\begin{tabular}{|c|c|c|c|c|c|c|c|c|c|c|}
\hline Factores de la seguridad vial & 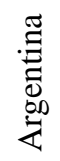 & $\stackrel{\stackrel{\pi}{ٍ}}{:}$ & $\begin{array}{l}\overline{\bar{z}} \\
\bar{\Xi} \\
\bar{n}\end{array}$ & $\frac{\frac{\pi}{0}}{\frac{\pi}{0}}$ & 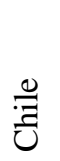 & $\begin{array}{c}\grave{0} \\
\stackrel{0}{0} \\
\tilde{J} \\
\tilde{I}\end{array}$ & 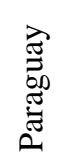 & 总 & 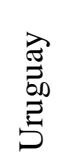 & 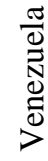 \\
\hline \multicolumn{11}{|l|}{ Gestión Normativa sobre seguridad vial } \\
\hline Organismo coordinador & Si & $\mathrm{Si}$ & $\mathrm{Si}$ & $\mathrm{Si}$ & $\mathrm{Si}$ & $\mathrm{Si}$ & $\mathrm{Si}$ & $\mathrm{Si}$ & $\mathrm{Si}$ & $\mathrm{Si}$ \\
\hline Participación de organismo en la elaboración PNSV & $*$ & $*$ & $*$ & $\mathrm{Si}$ & $*$ & $*$ & Si & $*$ & $*$ & $*$ \\
\hline Estrategia nacional & $\mathrm{Si}$ & $\mathrm{Si}$ & $\mathrm{Si}$ & $\mathrm{Si}$ & $*$ & $\mathrm{Si}$ & $\mathrm{Si}$ & $\mathrm{Si}$ & $\mathrm{Si}$ & $\mathrm{Si}$ \\
\hline Objetivos - metas & $\mathrm{Si}$ & $\mathrm{Si}$ & $\mathrm{Si}$ & $\mathrm{Si}$ & $\mathrm{Si}$ & $\mathrm{Si}$ & Si & $\mathrm{Si}$ & $\mathrm{Si}$ & $*$ \\
\hline Financiación (fondos) & $\mathrm{Si}$ & $\mathrm{Si}$ & $\mathrm{Si}$ & $\mathrm{Si}$ & $\mathrm{Si}$ & $\mathrm{Si}$ & $*$ & $*$ & $\mathrm{Si}$ & $*$ \\
\hline Indicadores de resultados y siniestralidad & $\mathrm{Si}$ & $\mathrm{Si}$ & $*$ & Si & $\mathrm{Si}$ & $*$ & $*$ & $*$ & $\mathrm{Si}$ & $*$ \\
\hline Estimación de la eficacia de las medidas en PNSV & $*$ & $\mathrm{Si}$ & $*$ & $*$ & $*$ & $*$ & $*$ & $*$ & $*$ & $*$ \\
\hline \multicolumn{11}{|l|}{ Vía de tránsito y movilidad más segura } \\
\hline Identificación de carreteras más peligrosas & $\mathrm{Si}$ & $*$ & $*$ & $\mathrm{Si}$ & $\mathrm{Si}$ & $\mathrm{Si}$ & $\mathrm{Si}$ & $*$ & $\mathrm{Si}$ & $*$ \\
\hline Evaluación (auditorias de seguridad vial) & $\mathrm{Si}$ & $\mathrm{Si}$ & $*$ & $\mathrm{Si}$ & $\mathrm{Si}$ & $*$ & $\mathrm{Si}$ & $\mathrm{Si}$ & $\mathrm{Si}$ & $\mathrm{Si}$ \\
\hline Capacitación transferencia de conocimientos & $\mathrm{Si}$ & $*$ & $*$ & $\mathrm{Si}$ & $*$ & $*$ & $\mathrm{Si}$ & $*$ & $*$ & $*$ \\
\hline Creación de nueva o mejora dela infraestructura viaria & $*$ & $*$ & $\mathrm{Si}$ & $\mathrm{Si}$ & $\mathrm{Si}$ & & $\mathrm{Si}$ & $\mathrm{Si}$ & $\mathrm{Si}$ & $*$ \\
\hline \multicolumn{11}{|l|}{ Vehículos más seguros } \\
\hline Inspección técnica de vehículos & $\mathrm{Si}$ & $\mathrm{Si}$ & $\mathrm{Si}$ & $\mathrm{Si}$ & $\mathrm{Si}$ & $\mathrm{Si}$ & $\mathrm{Si}$ & $\mathrm{Si}$ & $\mathrm{Si}$ & $\mathrm{Si}$ \\
\hline Equipamiento seguridad vehículos & $\mathrm{Si}$ & $*$ & $*$ & $*$ & & $*$ & $*$ & $*$ & $*$ & $*$ \\
\hline Reglamentación seguridad para vehículos a motor & $*$ & $*$ & $*$ & $*$ & $\mathrm{Si}$ & $*$ & $*$ & $\mathrm{Si}$ & $*$ & $*$ \\
\hline \multicolumn{11}{|l|}{ Usuario de las vías de transito más seguros } \\
\hline Velocidad máxima & $\mathrm{Si}$ & $\mathrm{Si}$ & $\mathrm{Si}$ & $\mathrm{Si}$ & $\mathrm{Si}$ & $\mathrm{Si}$ & $\mathrm{Si}$ & $\mathrm{Si}$ & $\mathrm{Si}$ & $\mathrm{Si}$ \\
\hline Vigilancia y cumplimiento normas & $*$ & $*$ & $*$ & $*$ & $\mathrm{Si}$ & $*$ & $*$ & $*$ & $*$ & $*$ \\
\hline Tiempos de Conducción & $\mathrm{Si}$ & $*$ & $\mathrm{Si}$ & $*$ & $\mathrm{Si}$ & $*$ & $\mathrm{Si}$ & $\mathrm{Si}$ & $\mathrm{Si}$ & $*$ \\
\hline Consumo de alcohol y conducción & $\mathrm{Si}$ & $\mathrm{Si}$ & $\mathrm{Si}$ & $\mathrm{Si}$ & $\mathrm{Si}$ & $\mathrm{Si}$ & $\mathrm{Si}$ & $\mathrm{Si}$ & $\mathrm{Si}$ & $\mathrm{Si}$ \\
\hline Uso del cinturón de seguridad & $\mathrm{Si}$ & $*$ & $\mathrm{Si}$ & $\mathrm{Si}$ & $\mathrm{Si}$ & $\mathrm{Si}$ & $\mathrm{Si}$ & $\mathrm{Si}$ & $\mathrm{Si}$ & $\mathrm{Si}$ \\
\hline Uso del casco en motocicletas y ciclomotores & $\mathrm{Si}$ & $\mathrm{Si}$ & $\mathrm{Si}$ & $\mathrm{Si}$ & $\mathrm{Si}$ & $\mathrm{Si}$ & $\mathrm{Si}$ & $\mathrm{Si}$ & $\mathrm{Si}$ & $\mathrm{Si}$ \\
\hline Uso de dispositivos de retención infantil & $\mathrm{Si}$ & $*$ & $\mathrm{Si}$ & $*$ & $\mathrm{Si}$ & $*$ & $*$ & $*$ & $*$ & $\mathrm{Si}$ \\
\hline Uso de teléfonos celulares al conducir & $\mathrm{Si}$ & $*$ & $\mathrm{Si}$ & $*$ & $\mathrm{Si}$ & $*$ & $\mathrm{Si}$ & $\mathrm{Si}$ & $\mathrm{Si}$ & $\mathrm{Si}$ \\
\hline Aumentar la concienciación & $\mathrm{Si}$ & $*$ & $\mathrm{Si}$ & $\mathrm{Si}$ & $\mathrm{Si}$ & $\mathrm{Si}$ & $\mathrm{Si}$ & $\mathrm{Si}$ & $*$ & $*$ \\
\hline Educación vial escuelas & $\mathrm{Si}$ & $*$ & $\mathrm{Si}$ & $\mathrm{Si}$ & $\mathrm{Si}$ & $\mathrm{Si}$ & $*$ & $\mathrm{Si}$ & $*$ & $*$ \\
\hline Formación vial-examen obtención licencia conducir & $\mathrm{Si}$ & $\mathrm{Si}$ & $\mathrm{Si}$ & $*$ & $\mathrm{Si}$ & $\mathrm{Si}$ & $*$ & $\mathrm{Si}$ & $\mathrm{Si}$ & $*$ \\
\hline Licencia por puntos & $\mathrm{Si}$ & $*$ & $\mathrm{Si}$ & $*$ & $*$ & $\mathrm{Si}$ & $*$ & $\mathrm{Si}$ & $*$ & $*$ \\
\hline Existencia de un registro único de conductores & $*$ & $\mathrm{Si}$ & $*$ & $*$ & $*$ & $*$ & $*$ & $*$ & $\mathrm{Si}$ & $*$ \\
\hline \multicolumn{11}{|l|}{ Respuesta tras los accidentes } \\
\hline Numero de atención para emergencias & $\mathrm{Si}$ & $\mathrm{Si}$ & $\mathrm{Si}$ & $\mathrm{Si}$ & $\mathrm{Si}$ & $\mathrm{Si}$ & $\mathrm{Si}$ & $\mathrm{Si}$ & $\mathrm{Si}$ & $\mathrm{Si}$ \\
\hline Sistema de atención pre hospitalaria & $*$ & $*$ & $*$ & $*$ & SI & $*$ & $*$ & $*$ & $*$ & $*$ \\
\hline Capacitación profesional & $\mathrm{Si}$ & & $\mathrm{Si}$ & $*$ & $\mathrm{Si}$ & $*$ & $\mathrm{Si}$ & $\mathrm{Si}$ & $*$ & $\mathrm{Si}$ \\
\hline Seguro obligatorio de accidentes de transito & * & $\mathrm{Si}$ & $\mathrm{Si}$ & $\mathrm{Si}$ & $\mathrm{Si}$ & $\mathrm{Si}$ & $*$ & $\mathrm{Si}$ & $\mathrm{Si}$ & $\mathrm{Si}$ \\
\hline
\end{tabular}

Tabla 3 - Factores marco metodológicos los PSV países América Sur. Elaboración propia

Respecto al segundo pilar: vías de tránsito y movilidad más segura Brasil, Ecuador y Venezuela son los países que tendrán que realizar mayor esfuerzo en este aspecto, los otros países han realizado sus primeras experiencias de una forma no sistemática y de manera ocasional. El tercer pilar: vehículos más seguros, todos los países tienen implementado la revisión técnica vehicular unos totalmente y otros en porcentajes. Casi todos los países presentan problemas en el equipamiento de seguridad de los vehículos excepto Argentina que tiene sus estándares de seguridad específicos para los vehículos. El cuarto pilar: usuarios de las vías de transito más seguros, el punto más débil es la vigilancia y el cumplimiento de las normas de tráfico en este sentido Chile es el único que cuenta con un centro automatizado de tratamiento de infracciones, otro aspecto es el uso de dispositivo de retención infantil tan 
solo Chile, Argentina, Brasil y Venezuela cuenta con una legislación y control de su utilización. De manera general se tiene que los países cuentan con una legislación que regula los aspectos fundamentales para conseguir usuarios más seguros pero el problema radica en hacer cumplir las normas. Y el último pilar: respuesta tras los accidentes, todos los países cuentan con un número de atención para emergencia, el problema radica en el sistema de atención pre-hospitalaria destacándose solo Chile al contar con este sistema. Después de abordar la problemática de la información y de la importancia de la evaluación dentro de los documentos de diagnóstico de seguridad vial realizado por la Asociación Española de Carretera y el BID, en la Tabla 4 se resumen algunas recomendaciones en común de las acciones a corto, mediano y largo plazo a ser implementadas en el cual es notorio nuevamente la mejora del sistema de recolecta de datos de siniestralidad y la elaboración de informes anuales y la inclusión de mejoras en la seguridad vial de los usuarios vulnerables como prioridad a corto plazo.

\begin{tabular}{|c|c|c|c|c|c|c|c|c|c|c|}
\hline Líneas de acciones sugeridas a ser implementadas & 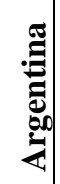 & $\begin{array}{l}\overline{\bar{s}} \\
\bar{s} \\
\bar{s}\end{array}$ & 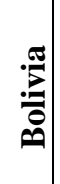 & $\begin{array}{l}\frac{\pi}{\hat{b}} \\
\frac{\mathrm{g}}{0} \\
\frac{0}{0}\end{array}$ & 窇 & 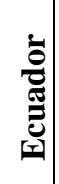 & 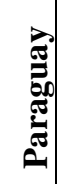 & & 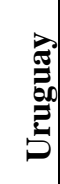 & 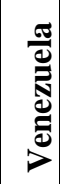 \\
\hline $\begin{array}{l}\text { Implantar sistemas de identificación y gestión de tramos de } \\
\text { concentración de accidentes }\end{array}$ & $\mathrm{C}$ & $\mathrm{M}$ & $\mathrm{M}$ & $\mathrm{C}$ & $\mathrm{C}$ & $\mathrm{M}$ & $\mathrm{M}$ & $\mathrm{L}$ & $\mathrm{M}$ & \\
\hline $\begin{array}{l}\text { Mejorar sistemas de recoleta de dados de siniestralidad y } \\
\text { elaborar informes anuales }\end{array}$ & & $\mathrm{C}$ & $\mathrm{C}$ & $\mathrm{C}$ & & $\mathrm{C}$ & $\mathrm{C}$ & $\mathrm{C}$ & C & $\mathrm{C}$ \\
\hline $\begin{array}{l}\text { Incluir mejora en la seguridad vial de los usuarios vulnerables } \\
\text { como prioridad }\end{array}$ & & $\mathrm{C}$ & $\mathrm{C}$ & $\mathrm{C}$ & $\mathrm{C}$ & $\mathrm{C}$ & $\mathrm{C}$ & $\mathrm{C}$ & $\mathrm{C}$ & $\mathrm{C}$ \\
\hline Fortalecer el control del cumplimiento de las normas & $\mathrm{C}$ & $\mathrm{C}$ & $\mathrm{C}$ & $\mathrm{M}$ & & $\mathrm{C}$ & $\mathrm{M}$ & $\mathrm{C}$ & $\mathrm{C}$ & $\mathrm{C}$ \\
\hline Aplicación de inspecciones y auditorías de seguridad vial & $\mathrm{C}$ & & & $\mathrm{C}$ & & & & & & \\
\hline Garantizar la existencia de profesionales de la seguridad vial & & $\mathrm{M}$ & $\mathrm{M}$ & $\mathrm{M}$ & $\mathrm{L}$ & $\mathrm{M}$ & $\mathrm{M}$ & $\mathrm{M}$ & $\mathrm{M}$ & $\mathrm{M}$ \\
\hline Enseñanza de la seguridad vial en escuelas & & $\mathrm{M}$ & & & & & & & $\mathrm{M}$ & $\mathrm{M}$ \\
\hline Promover la coordinación regional en materia de seguridad vial & & $\mathrm{L}$ & $\mathrm{M}$ & $\mathrm{L}$ & & $\mathrm{M}$ & $\mathrm{L}$ & $\mathrm{L}$ & $\mathrm{L}$ & $\mathrm{L}$ \\
\hline $\begin{array}{l}\text { Apoyar la introducción de estándares de seguridad en los } \\
\text { vehículos automotores }\end{array}$ & & $\mathrm{L}$ & $\mathrm{M}$ & $\mathrm{M}$ & & $\mathrm{M}$ & $\mathrm{M}$ & $\mathrm{M}$ & $\mathrm{M}$ & $\mathrm{M}$ \\
\hline $\begin{array}{l}\text { Alentar actividades de investigación y desarrollo de la } \\
\text { seguridad vial }\end{array}$ & $\mathrm{L}$ & & $\mathrm{L}$ & $\mathrm{L}$ & $\mathrm{L}$ & $\mathrm{L}$ & & & $\mathrm{L}$ & \\
\hline Incluir la asistencia médica tras los accidentes & & $\mathrm{L}$ & $\mathrm{L}$ & & & $\mathrm{L}$ & & $\mathrm{L}$ & $\mathrm{L}$ & $\mathrm{L}$ \\
\hline Aumentar el porcentaje de red viaria pavimentada & & $\mathrm{L}$ & & $\mathrm{L}$ & & & & $\mathrm{L}$ & & \\
\hline Fortalecer una agencia líder de seguridad vial & & & $\mathrm{C}$ & & & $\mathrm{C}$ & & & & \\
\hline Crear una agencia líder de seguridad vial & & & & & & & & & & $\mathrm{C}$ \\
\hline Centro de tratamiento automatizado de infracciones & & & & & $\mathrm{C}$ & & & & & \\
\hline
\end{tabular}

Tabla 4 - Acciones seguridad vial sugerida para los países de América Sur. Elaboración propia

\section{DISCUSIÓN Y CONCLUSIONES}

Cualquier plan de mejora de la seguridad vial realizado por los países de América del Sur requiere el estudio riguroso y un amplio conocimiento de las causas de los accidentes de tránsito y contar con un modelo evaluación riguroso de las diferentes estrategias adoptadas en materia de seguridad vial para verificar el cumplimiento de los indicadores propuestos y estimar los efectos de las acciones sobre accidentes tránsito.

Pero la información necesaria, no está estructurada de manera adecuada y su utilidad es limitada y escasa para el trabajo de investigación, análisis y diagnóstico de la situación y la 
adopción de medidas de mejora en seguridad vial, adaptadas a la situación de cada país.

Si bien todos los países cuentan con un diagnóstico de la situación de los accidentes de tránsito y los esfuerzos por disminuirlos, no pueden ver logros significativos por el problema real de la información escasa y desestructurada antes señalado. En el análisis regional destacan los países de Argentina y Chile los cuales presentan una situación de liderazgo en América del Sur y pueden ser ejemplos a seguir para los otros.

Sin embargo, las acciones guiadas por imitaciones o adopción de prácticas de éxito en un país puede perfectamente resultar un fracaso cuando se aplica en otro entorno sin tener en cuenta las particularidades nacionales o regionales y la necesidad de valorar el componente cultural.

A pesar de la aprobación de diversas regulaciones sobre tránsito y seguridad vial, no se observa una tendencia hacia la disminución de las lesiones fatales en los usuarios del tránsito.

La problemática de la información presenta dificultades para reconocer la real situación y ocasiona enormes problemas para establecer comparaciones entre países dentro de la región, en consecuencia, se puede decir que se desconoce la real magnitud del problema, sus impactos y los costos asociados, en términos económicos, de bienestar, calidad de vida y desarrollo, haciendo poco objetiva la formulación de políticas públicas y de la misma forma la evaluación de las mismas.

En el corto plazo, en algunos países y en tanto que se implementan medidas eficaces de recogida, estructuración y análisis de la información, y se cuente con series históricas que permitan aplicar métodos correlacionales para efectuar análisis "macro" de la situación accidentológica a niveles nacionales o regionales dentro de determinados países, puede ser útil complementar la información disponible con la aplicación de métodos cualitativos como los que permiten identificar, recoger y sistematizar el conocimiento experto de especialistas, responsables de la gestión del tránsito y otros agentes involucrados para, en base a dicho conocimiento, adoptar decisiones encaminadas a mejorar las condiciones generales de seguridad vial y reducir el número de accidentes y daños ocasionados por los mismos.

\section{REFERENCIAS}

AGENCIA NACIONAL DE SEGURIDAD VIAL. (5 de 10 de 2010). Agencia Nacional de Seguridad Vial Disposición 294/2010. Recuperado el 2015, de http://concursos.seguridadvial.gov.ar/pdf/plan_control.pdf ANT. (23 de 8 de 2013). Agencia Nacional de Tránsito . Obtenido de http://www.obraspublicas.gob.ec/wpcontent/uploads/downloads/2013/10/DIA1_02_ANT_Plan_Nacional_Seguridad_Vial.pdf CONASET. (2 de 2 de 2013). Obtenido de https://www.mtt.gob.cl/wpcontent/uploads/2014/02/conaset.pdf

CONSEJO NACIONAL DE SEGURIDAD VIAL. (1 de 7 de 2013). Consejo Nacional de Seguridad Vial. Obtenido de www.mopc.gov.py/mopcweb/pdf/pnsv_2008_2013.pdf DENATRAN. (6 de 5 de 2011). DENATRAN - Departamento Nacional de Trânsito. Obtenido de http://www.denatran.gov.br/download/decada/Proposta\%20ANTP-CEDATTInstituto\%20de\%20Engenharia\%20SP.pdf

ELVIK, R. (2008). Dimensions of road safety problems and thier measurement. Accident Analysis and Prevention, 40:1200-1210.

EVO, M., CHOQUEHUANCA, D., QUINTANA, J., JORGE, P., SAAVEDR, R., CARO, 
E., ... Hernando, J. (13 de 8 de 2014). Gaceta Oficial del Estado Plurinacional de Bolivia. Obtenido de http://www.gacetaoficialdebolivia.gob.bo

HADDON, W. (1972). A logical framework for categorizing highway safety phenomena and activity. Journal of Trauma and Acute Care Surgery, 12(3), 193-207.

IZQUIERDO, J. D., \& TORRES, R. (2009). Hacia una sociología de la seguridad vial: del factor humano al " factor social" . Praxis sociologia, 13:148-172.

MINISTERIO DE TRANSPORTE DE COLOMBIA. (14 de 08 de 2014). Ministerio de Transporte de Colombia. Recuperado el 2 de 22 de 2016, de https://www.mintransporte.gov.co/descargar.php?idFile=11562

MINISTERIO TRANSPORTE Y OBRAS PÚBLICAS. (2008). Ministerio Transporte y

Obras Públicas. Obtenido de

http://www.oisevi.org/a/archivos/normativas/uruguay/Uruguay_UNASEV_Plan_Nacional _de_Seguridad_Vial_2008-2012.pdf

MINISTRO DE TRANSPORTES Y COMUNICACIONES. (5 de 1 de 2015). Ministro de Transportes $y \quad$ Comunicaciones. Obtenido de https://www.mtc.gob.pe/cnsv/Proyecto\%20del\%20Plan\%20Nacional\%20de\%20Seguridad \%20Vial\%202015_2024.pdf

OISEVI. (sf de sf de 2015). OISEVI. Obtenido de http://www.oisevi.org/a/index.php/ ONU. (10 de Mayo de 2010). Organizacion Naciones Unidas. Recuperado el 6 de Noviembre de 2015, de http://www.un.org/es/comun/docs/?symbol=A/RES/64/255f ORGANIZACION MUNDIAL DE LA SALUD. (2009). Informe sobre la situación mundial de la seguridad vial: es hora de pasar a la acción. Ginebra, Suiza: Organización Mundial de la Salud.

ORGANIZACION MUNDIAL DE LA SALUD. (2013). Organizacion Mundial de la Salud. Recuperado el 14 de 11 de 2015, de http://www.who.int/violence_injury_prevention/road_safety_status/2013/es/ ORGANIZACIÓN PANAMERICANA DE LA SALUD. (2009). Informe sobre el Estado de la Seguridad Vial en la Región de las Américas. Washington, D.C.:: OPS.

SIVAK, M., \& TSIMHONI, O. (2008). Improving traffic safety: conceptual considerations for successful action. Journal of Safety Research, 39:453-7. 